\title{
Nene Bestimmung der Meereshöhe von Marburg.
}

Die Angabe in Nr. 458 S. 27 grïndete sich bekanntlich der Hauptsache nach auf einseitige Barometerbeobachtungen, indem sich danals noch keine bessere Hülfsmittel fanden. Diese hahen sich seitden gefunden, einestheils in einem System von zuverlässigen Zenithdistanzen, welches jetzt zwischen der Göttinger Sterwwarte und meinem hiesigen Beobachtungszimmer auf dem Thurm des Dörnberger Hofs besteht, anderntheils in den Wasserbau - Nivellirungen, welche jetat von $\mathbf{A ~ m}$. sterdam aus bis in die Lahn bei Marburg ununterbrochen sich erstrecken.

Da die durch solche Hülfsmittel gewonnenen Zahlen, selbst wenn sie weniger genau zusammenstimmten als wirklich der Fall ist, (auf 0,5 par. Fufs) und wenn sie weniger als sich dabci herausstellt ( 43 par. F.) von jener Barometerbestimmung abwichen, doch letztere nunmehr ganz zu verwerfen gebieten würden; so möchte es vielleicht genügend erscheinen, blofs die verbesserte Zahl anzugeben. Ich erlaube mir aber die Mittelstufen, welche dazu fübrten etwas ausführlicher darzustellen, weil sie ein Bild von dem geben, was die gegenwärtigen Methoden für einen Punkt des Binnenlandes leisten, welcher auf beiden vielfach gekrümmten Wegen nahe $60 \mathrm{Mei}$ len vom Meere entfernt liegen mag, und weil mehrere Zwischenpunkte dabei vorkommen, welche auch für sich schon ein Interesse gewähren. Vielleicht könnte auch dadurch Ver. anlassung gegeben werden einzelne Elemente noch weiter zu prüfen.

I. Bei der trigonometrischen Bestimmung durch Zenithdistanzen liegt

1) der Fufsboden der Gïtinger Sternoute zum Grunde. Seine Neereshöhe ist abgeleitet aus den Zenithdistanzen, welche bei der hannoverschen Gradmessung heobachtet wurden und sich bei Langwerden im Butjadinger Land an die Nordsee anschlossen. Dic von Gaufs selbst dafür gefundene Zahl ist 155,836 Walbecksche Meter, die mit dem Verwandlungs - Iogarithnus 9,7101917 in Toisen übersetzt werden. Dies giebt

$$
a=479,74 \text { pariser liufs. }
$$

2) Hieran schliefsen sich die, unter der Direction des Herrn Obristlieutenant Wiegrebe stehenden topographiscben Messungen in Kurhessen. Dieselhen sind auf den durch meine Triangulirung gewonnenen Grundrifs gebaut, und haben sich ihrem trigonometrischen Theile nach schon vor zwei Jahren bis Marburg erstreckt, während in diesem Jahre hier schon mit Mefstischen gearbeitet wird. Nach allem was ich davon bisjetzt geschen, wird uichts versäunt, was ihnen den Rang unter den besten Arbeiten dieser Art sichern könnte. Namentlich ist ein ganz vollständiges, durchweg geprüftes und ausgeglichenes System gegenseitiger und für Hauptpunkte auch gleichzeitiger Zenithdistanzen dahei eingeführt. So wurde denn auch mein Dörnberger Hof-Thurm von drei henachbartrn Bergen aus mit dem obigen (Göttinger Fufshoden aufs sicherste verbunden. Ich selbst leistete dazu keinen weiteren Beitrag, als die dem Herrn Geometer Coester gewährte Beihülfe in gevauer Ablithung der Spitze des Thurms auf den Fufsboden des Beohachtungszimmers. Schon inı vorigen Sommer erbielt ich aus diesen Operationen für die Höhe meiner in Nr. 458 erwähnten Plattforn ( = Fufshoden des Beolachtungsximmers) über dem Göltinger Fursboden, die Zahl 24,60 preufssische Ruthen, welche mit dem Verwandl. Log. J,0642395 in par. Fuls übersetzt werden. Dies giebt

$$
b=285,24 \text { p. F. }
$$

Folglich ist die trigonometrische Bestimmung der gesuchten verbesserten Meereshöhe

$$
A=a+b=764,98 \text { p. F. }
$$

II. Die Bestimmung aus Nivellirungen ergiebt sich aus der folgenden Zusammenstellung.

1) Durch die Gïte des Hern Geheimerath Hagen in Berlin erhiclt ich in diesen letzten Frubjahr eine Uebersicht der sämmtlichen Wasserbau-Niveliirungen von den $Y$ bei A nusterdam bis zu dem Wehr-Rücken (Wehrfach = OberWasser) der Wasserkunst und s. g. Papiermühle in der Lahn bei Marburg, welcher in der Umgebung von Marburg als Nullpunet der Nivellirungen dient. Ich lasse also Herrn Hagen's Original-Angalien hier folgen, indem ich nur zwei kleine Nachträge gleich einschalte, deren einer auf seine Ver. anlassung von Herrn Wasserhau. Inspector Asmus in Wetzla r mir mitgetheilt wurde, während der andere von ihm selbst herrïhte.

x) Der Nullpunkt des Pegels bei Capellen, der Mündung der Lahn gegenüber, liegt 172,73 preufsische Fufs zu 139,13 par. Linien über Amsterdammer Peil, d. h. üher der mittleren Fluthhöhe vom $Y$. - Der mittlere oder gewölın. liche Wasserstand des Rheins vor der Mündung der Lahn, von welchem die nächstfolgende Angabe angenommen wird, ist nahe 7 Fufs über jenem Nullpunkt. Eine schärfere Angabe lärst sich demnächst noch vielleicht ernitteln, vor der Hand aber ist der Ausgangspunkt der folgenden Angabe zu setzen in runder $\mathrm{Zahl}$

$$
=180 \text { preurs. Furs. }
$$

B) Gefalle der Lahn in Nassauischen 244,041 nassauische Fufs zu 0,3 Meter

$$
=233,3 \text { pr. F. }
$$


r) Desgleichen auf der preufsischen Strecke, nach Abzug von 1,37 pr. F., welche auf dem rechten (preufsischen) und auch auf dem linken (nassauischen) Ufer, also doppelt, gemessen waren

$$
=56,23 \mathrm{pr} . \mathbf{F} \text {. }
$$

d) Desgleichen im grofsherzoglich hessischen Gebiet 46,96 hessische Fufs zu 0,25 Meter

$$
=37,4 \text { pr. F. }
$$

в) Desgleichen im kurfürstl. hessischen Gebiet von jenem oben erwähnten Nullpunkt aus $55^{\prime} 8^{\prime \prime} 8^{\prime \prime}$ nach Kasseler Normalmaafs den Fufs zu 11 rheinl. Zoll gerechnet

$$
=51,1 \text { pr. F. }
$$

Diese fünf Gefälle zusammenaddirt geben also 558,03 preussische Fufs fïr die Meereshöhe des Nullpunkts in der Lahn, und also

$$
c=539,16 \text { pariser Furs. }
$$

2) Meine Nivellirungs-Operationen bezogen sich nun auf zwei verschiedene Punkte der Lahn-Nivellirung die beide von jenem Nullpunkt versclieden sind. Ich erhielt über diese beiden Punkte rom Herrn Wasserbaumeister Bauer hieselbst folgende Angaben:

$d=$ Fach des Lohmühlenwehrs in Mühlgraben der Papiermühle und Wasserkunst 5'6"9"'35 unter Null.

$e=$ Scheitel der innern Bogenlinie des mittleren Bogens der Elisabeth-Brücke 18'8" $5^{\prime \prime \prime} 2 j$ über Null.

Dieses giebt nach dem angeführten Verbältuís reducirt

$$
\begin{aligned}
& d=4,93 \text { par. } \mathbf{F} \text {. } \\
& e=16,57
\end{aligned}
$$

3) Die Messungen selbst bestehen nun aùs zwei von einander durchaus unabhängigen Operationen. Beide sind mit derselben Nivellirlatte gemacht, welche eigentlich Kurhessische Katasterruthen zu $14.126,3$ pariser Linien vorstellen solite. Dieselbe war aher angefertigt lange ehe ich in den Besitz einer Normal-Toise kam. Deshalb habe ich sie jetat vor einigen Wochen erst surgfältig mit der Toise verglichen und gefunden, dafs sie ihr Maafs nicht ganz gemau darstellt, sondern dafs sich diese Kuthen mit dem Verw. Log. 1,0894913 in pariser Fufs ïbersetzen.

a) In meinem Nr. 458 angefübrten Schriftchen S. 147 habe ich angegehen, dafs der erste Barometerplatz meiner früheren Arbeit 15,4027 solcher Ruthen über dem vorstehend erwähnten Lohmühlenwebr schon 1827 gefunden war. Die Messung, welche ich im Winter $184 \frac{1}{2} \mathrm{mit}$ dem seel. Westphalen ausführte, bestand nun darin, dafs wir zuerst von Fen. ster zu Fenster einen Fufsboden des jetzigen Hauses über jenem Barometerplatz nivellirten und sodann den verticalen Abstand desselben von dem liufsboden des Beobachtungszimmers des Thurms, welcber zu diesem und ähulichen Zwecken durchlöchert ist, an einem hängenden Metalldrath un- mittelbar abmaafsen. Dadurch ergab sich letzterer Fufsboden 3,3428 Ruthen höher als jener Barometerplatz, und also die zu bestimmende Ebene 18,7455 Ruthen üler dem Lohmühlenwehr, d. h. nach Obigem

$$
f=230,35 \text { par. F. }
$$

B) Da die Nivellirung von 1827 nur auf einem einzelnen, obwobl sorgfaltig bearbeiteten Zug beruhte, so begann ich im Herbst 1844 die zweite Messung. Ich stellte nämlich das Nivellirinstrument auf den fraglichen Fufsboden und bestimmte dadurch seine Höhe über einem Pflock der in etwa 350 Ruthen Entfernung am Berge jenseits der Lahn cingeschlagen war, stellte sodann, mit Zurücklassung eines verticalen Maafsstabs das Instrument am Berge in der Nähe desselben auf, und maafs so dieselbe Grüfse rïckwärts. Das Mittel gab: Fufsboden über Pflock 0,5237 Ruthen. In Frühjahr 1845 wiederholte ich dieselbe Operation in umgekehrter Ordnung und fand 0,5299 Ruthen, so dafs ich im Mittel 0,5268 ansetzen konnte. Eine, mit Hïlfe des Hrn. Hrndschuh, am Berg hinunter geführte Nivellirung gab nun ferner durch 22 Aufstellungen jenen Pflock über dem zu 2) erwähnten Brückenbogen 16,4449. Dieses mit obigem Mittel zusammengenommen giebt also die Höhe des fraglichen Fufsbodens über dem Brückenbogen 16,9717 luthen, oder nach obiger Reduction

$$
g=208,55 \text { par. Furs. }
$$

Demnach fand sich fïr die Höhe über dem Nullpunkt der Lahn

$$
\begin{aligned}
\text { aus } x) f-d & =225,42 \text { p. F. } \\
\beta) g+e & =225,12
\end{aligned}
$$

und kann ich wohl als definitiv das Mittel aus beiden Opera. tionen ansetzen

$$
h=225,27 \text { p. F. }
$$

Die Bestimmung aus Nivellirungen giebt also

$$
B=c+h=764,43 \text { p. F. }
$$

In Nlittel aus den beiden Bestimmungen $A$ und $B$ habe ich also statt der Nr. 458 gegebenen Zahl 808 nunmehr zu setzen 765 pariser Fufs.

Dürfte man nun das überraschend genaue Zusammenfallen der beiden verschiedenen Bestimmungen lür mehr als einen besonders glïcklichen Zufall halten, so würde daraus zunäcbst zu folgern seyn, dafs der Ausgangspunct der Gaussischen Zenithdistanzen mit Amsterdammer Peil übereinstimmte. Von diesem Ausgangspunkt ist mir durch briefliche Mittbeilung nur bekannt, dafs die Bestimmung sich auf den Spiegel der Nordsee "nicht bei niedrigem Wasser, sondern vielleicht eher bei hohem Wasser" bezieht.

Dagegen erfuhr ich, dafs Nivellirungen für die Eisenbahn, welche von dem Nullpunkt am Pegel der grofsen Harburger 
Scbleuse ausgingen, diesen (nach einer Angabe des seel. Woltmann; $t^{\mathrm{m}} 152$ höher setzten als das ordinäre niedrige Wasser hei Cuxhafen, und endlich danit den Göttinger Fulsboden 16 pariser Fufs hüher fanden als die obige $\mathrm{Zahl} A$ angiebt.

Marburg 1846. October 9
Die Frage nach der Genauigkeit der obigen Zahl für meine verbesserte Meereshöhe würde also zunächst auf eine Frage nach dem Meereszustand anden Küsten der Nordsce zurückkommen, über welchen mir bisjetzt nichts bekannt ist.

\section{Preise meiner Chronometer und Pendeluhren.}

Astronomische Pendelubren mit Rost- oder Quecksilberpendel, so eingerichtet, dafs man das Gangrad und den Ha. keo herausnehmen kann, ohne das Werk ganz auseinander zu nehmen .................. 300 Rthlr.

Dieselben Uhren in grörserer Vollkommenheit ausgeführt. 400 Rthlr.

Box-Chronometer die 56 Stunden gehen und halbe Se. cunden schlagen, mit einem Zeiger der die Zeit angiebt, welche seit dem Aufzichen verflossen ist ....... 300 Rthlr.
Dieselben 8 Tage gebend .......... 450 Rthlr.

Auf besondere Bestellung Taschen-Chronometer in sil. bernen Gehäusen ................ 350 Rthlr.

Dieselben in goldenen Gehäusen ......4 400 Rthlr.

Berlin 1847.

Tiede.

$$
V \text { e r m is ch } t \text { e Nach } \quad \text { ich } t \text { e u. }
$$

Herr Dr. Julu in Leipzig hat mich ersucht anzuzeigen,

1) dafs ein Verzeichnil's aller bis zum Jahre 1847 berechneten Cometenbahnen von ihm zusammengetragen, bald im Verlage von Breithopf und Hertel erscheinen werde;

2) dafs seit dem $2^{\text {ten }}$ Januar $\mathbf{d}$. J. die von ihtn redigirte Zeitschrift,

Wöchentliche Unterhaltungen für Dileltanten und Frcunde der Astronomie, Geographie und Witterungskunde, im Verlage von Rob. Friese in Leipzig erscheine. Es wird wöchentlich eine Nummer von einem halben Bogen in $8 v 0$ ansgegeluen. Der Preis des Jahrganges ist $2 \frac{2}{3}$ Thal. Pr.

\section{I $\mathbf{n}$ h a $\mathbf{l}$ l t.}

(Zu Nr. 601). Cometenbeobachtungen anf der Berliner Sternwarte p. 1. Beobachlungen der Astraea und des Neptnn in Kasan p. 6.

Beobachtungen der Astraea alif der Hamburger Sternwarte p. 9.

Osservazioni della Cometa di Hind del 6 Febbrajo 1847 fatte nell J. R. Osservatorio di Padova p. 9. -

Neue Bestimmung der Meereshöhe von Marburg p. 11. -

Preise der Clrronometer und Pendeluhren von Tiede p. 15. -

Vermischte Nachrichten p. 15. -

Altona 1847. Juni 14. 